\title{
Personalizing prognosis in a patient with serious illness
}

\author{
Jeff Myers MD MSEd, Debbie Selby MD
}

A ddressing prognosis for a patient with a serious or progressive, life-limiting illness presents a unique challenge. What a person understands about his or her illness and what he or she expects to occur substantially affects both the decisions made regarding care and the overall outcomes. ${ }^{1}$ Because rapid growth is expected in this patient population, developing and improving communication skills and engaging in meaningful discussions to address prognosis will become increasingly important for clinicians. In this commentary, we explore the challenges clinicians face in addressing prognosis for a patient with a progressive, lifelimiting illness, and we propose for clinicians an approach to framing and conducting a discussion that addresses prognosis.

Expanding the concept of prognosis is essential. Prognosis includes an estimation of the disease course, and the possible impact of both the disease and the treatments being considered. In the clinical setting, however, "prognosis" is often used to represent an estimate of the patient's likely survival duration, which is a small component of what the term broadly encompasses. ${ }^{2}$ When understood in this way, prognostic disclosure becomes a unidirectional process whereby a clinician provides only a survival estimate to the patient. A critical limitation of this process is that survival estimates tend to be highly inaccurate.,.4 To date, the primary focus for advancing the science of prognosis has been on improving the accuracy of survival estimation. However, one could argue that, no matter how successful the effort to develop state-of-the-art models for estimating survival, estimates are likely to be inaccurate and carry a risk of patients being misinformed. A different approach to prognosis is needed.

Clinicians must learn to reframe prognostic disclosure as an individualized, patient- and family-centred prognostic discussion. Analogous to this concept is personalized medicine and the use of molecular data from a patient to inform diagnostic and therapeutic processes, which enables increased specificity and sensitivity for both disease classification and targeted therapies. ${ }^{5}$ For a patient with progressive, life-limiting illness it is equally important to arrive at a "personalized prognosis," drawing on person-specific data to inform an individualized prognostic discussion. The discussion should be a dynamic and iterative exchange of information and involve more than simply a one-time estimate of survival. We cannot overstate that this is an advanced communication process that must be highly individualized.

The approach to a prognostic discussion must involve two elements: exploring both the patient's understanding of their illness as well as their goals of care. This exchange of information should result in the patient being able to prioritize his or her goals of care. This can occur only if a patient can contemplate his or her values and beliefs, having understood the individualized illness-related information they consider meaningful for the current context. The content and function of the information provided by the clinician, as well as the best method of presenting the information, is determined through a process of assessing what the patient knows, what he or she believes they need to know and how the information might facilitate decision-making for any given context. A survival estimate may not be the prognostic information a patient needs. Instead, the patient may need to be asked questions like "What's the most important thing to you?" or "What do I need to know about you to provide the best care possible?" Responses to such questions may help a clinician facilitate the process of prioritizing goals of care.

What are the specific goals of care that require prioritization? Patients describe goals of

\section{KEY POINTS}

- The future health care landscape will include a growing population of patients living with progressive, life-limiting illness.

- The current approach to prognosis for these patients is often a unidirectional communication of a survival estimate that is often inadequate, because estimates are highly inaccurate and patients' values are not considered.

- Prognosis must be framed as an iterative and dynamic exchange of information that results in a patient being able to prioritize his or her goals of care.

- Decisions about care preferences must be informed by prioritized goals of care and reflect the patient's values and beliefs within the current context.

- Widespread acquisition of skills enabling person-centred prognostic discussions must occur among clinicians in both acute and ambulatory settings.

All editorial matter in CMAJ represents the opinions of the authors and not necessarily those of the Canadian Medical Association. 
care in broad themes that may coexist: to be cured, to improve and maintain quality or function, to be comfortable, to live longer, to achieve life goals or to provide support to family. ${ }^{6}$ The patient's goals of care may not include the goals clinicians consider, for example resuscitation interventions or code status. The challenge for a clinician is to collaborate with the patient and translate information into decisions regarding care preferences, while ensuring each preference is an accurate reflection of the patient's values and beliefs within the current context.

What does a patient understand about his or her illness? Multiple reports suggest a high prevalence of inaccurate understanding among patients with a serious illness. ${ }^{1,7}$ An element of this multidimensional construct includes patients not appreciating either the incurable or progressive nature of their illness. In a study by Allen and colleagues, ${ }^{7}$ patients with congestive heart failure were asked, "What do you think the eventual outcome will be from your heart failure?" Only 36\% indicated they understood "it would likely shorten their life." Similarly, in a study involving patients with metastatic non-small-cell lung carcinoma, at baseline 35\% accurately understood that their illness was not curable and $31 \%$ accurately understood that chemotherapy would not eliminate their cancer. ${ }^{1}$ Patients in the study arm received early palliative care as an intervention and were significantly more likely to maintain or gain an accurate understanding of both curability and the goal of therapy. Their understanding was subsequently found to be associated with certain treatment decisions near end of life. The relation between accuracy of illness understanding and the process of prioritizing goals of care remains unknown. A potential consequence of an inaccurate illness understanding is a patient giving consent to specific treatments that might otherwise have been declined.

Studies addressing the information needs of patients related to understanding of illness have consistently reported that patients desire detailed information, certain types of information (e.g., quantitative or qualitative) and information that is both optimistic or hopeful and realistic or practical. ${ }^{8,9}$ Information needs vary greatly between patients and over time, which highlights the importance of continually discussing them with a patient.

For a patient with a progressive, life-limiting illness, discussions that address understanding of illness and goals of care could begin as early as the initial diagnosis or shortly thereafter. This timing broadens the scope of professional relevance and requires that all clinicians - in both acute and ambulatory care settings - acquire skills that enable person-centred prognostic discussions. The related development of communication skills should be considered a key competency. Given the limited number of palliative care clinicians, their time might be wisely invested in educating and mentoring current and future clinicians. As a profession, we must work toward the capacity to arrive at a meaningful, personalized prognosis for each person living with a progressive and life-limiting illness.

\section{References}

1. Temel JS, Greer JA, Admane S, et al. Longitudinal perceptions of prognosis and goals of therapy in patients with metastatic non-small-cell lung cancer: results of a randomized study of early palliative care. J Clin Oncol 2011;29:2319-26.

2. You JJ, Fowler RA, Heyland DK. Just ask: discussing goals of care with patients in hospital with serious illness. CMAJ 2013 July 15; [Epub ahead of print].

3. Selby D, Chakraborty A, Lilien T, et al. Clinician accuracy when estimating survival duration: the role of the patient's performance status and time-based prognostic categories. J Pain Symptom Manage 2011;42:578-88.

4. Hanahan D, Weinberg RA. Hallmarks of cancer: the next generation. Cell 2011;144:646-74.

5. Glare PA, Sinclair C. Palliative medicine review: prognostication J Palliat Med 2008;11:84-103.

6. Haberle TH, Shinkunas LA, Erekson ZD, et al. Goals of care among hospitalized patients: a validation study. Am J Hosp Palliat Care 2011;28:335-41.

7. Allen LA, Yager JE, Funk MJ, et al. Discordance between patient-predicted and model-predicted life expectancy among ambulatory patients with heart failure. JAMA 2008;299:2533-42.

8. Innes S, Payne S. Advanced cancer patients' prognostic information preferences: a review. Palliat Med 2009;23:29-39.

9. Howlett J, Morrin L, Fortin M, et al. End-of-life planning in heart failure: It should be the end of the beginning. Can J Cardiol 2010; 26:135-41.

Affiliations: Division of Palliative Care (Meyers), Department of Family and Community Medicine, University of Toronto; Palliative Care Consult Team (Selby), Sunnybrook Health Sciences Centre, Toronto, Ont.

Contributors: Both authors contributed substantially to developing the concept, writing and editing of the manuscript. 